\title{
Sensivity Analysis of the Cellular Automata Model for Austenite-Ferrite Phase Transformation in Steels
}

\author{
Rafal Golab*, Daniel Bachniak, Krzysztof Bzowski, Lukasz Madej \\ AGH University of Science and Technology, Kraków, Poland \\ Email: ‘rgolab@agh.edu.pl,bachniak@agh.edu.pl,kbzowski@agh.edu.pl,1madej@agh.edu.pl \\ Received September 18, 2013; revised October 18, 2013; accepted October 25, 2013
}

Copyright (C) 2013 Rafal Golab et al. This is an open access article distributed under the Creative Commons Attribution License, which permits unrestricted use, distribution, and reproduction in any medium, provided the original work is properly cited.

\begin{abstract}
The main goal of the present research is to realize a sensitivity analysis of the developed complex micro scale austenite $(\gamma)$ to ferrite $(\alpha)$ phase transformation model. The proposed solution is implemented in the developed Cellular Automata Framework that facilitates implementation of various microstructure evolution models. Investigated model predicts phase transformation progress starting from the fully austenitic or two-phase regions. Theoretical background of the implemented austenite-ferrite phase transformation model is presented in the paper. The defined transition rules for initiation and subsequent growth as well as internal variables for each particular CA cell are also discussed. Examples of results obtained from the developed model, as well as model capabilities are shown. Finally sensitivity analysis using Morris OAT Design is also presented and discussed.
\end{abstract}

Keywords: Sensitivity Analysis; Cellular Automata; Austenite; Ferrite; Phase Transformation

\section{Introduction}

Small, medium and large industrial companies aim to develop an innovative and efficient manufacturing technology to make products that are competitive on the global market. However, development of these new technologies based on conventional approach composed of industrial test and trials is very expensive and time consuming. As a result, industry cannot quickly adapt to the rapidly changing demands of the market. Thus, recently, industry more often relies on technology development supported by a series of numerical calculations and computer simulations. These approaches provide possibility to accurately describe material behavior not only at the macro scale level but also can predict changes in the microstructure at the mezo or micro scale levels. As a result, significant drop in the amount of laboratory or industrial tests is possible. This creates an opportunity to save valuable time and also decrease R\&D costs. However, access to appropriate hardware and software is crucial in this approach; otherwise it is impossible to take full advantage of the computer aided technology design.

Conventional numerical approaches are usually based on the finite element method (FEM) combined with standard closed-form micro scale models that can provide

\footnotetext{
"Corresponding author.
}

general information about the state of the material e.g. temperature, average phase volume fraction, grain sizes, stress-strain state etc. However, exact information on material microstructure and morphology is also crucial when development of novel manufacturing technology is undertaken by a company. Unfortunately, these fast conventional models fail to provide such detailed data. The solution to this limitation is replacing the conventional models with modern discrete models based on e.g. Monte Carlo (MC), Cellular Automata (CA) etc. The CA model besides standard information on material behavior can also provide information about the final morphology of the material after, but also during, the process as shown Figure 1.

In the last decade, series of microstructure evolution models based on the cellular automata method were created [1-4]. Unfortunately, these models were designed as in-house codes and have the capability to solve only selected problem e.g. the simulation of dynamic or static recrystallization. If a new $\mathrm{CA}$ model e.g. austenite to ferrite phase transformation is required, the algorithmic and programming work has to be initiated from scratch and that significantly rises model development time.

To solve this issue, authors decided to develop a universal framework for the cellular automata method. All the basic cellular automata algorithmic solutions are avail- 

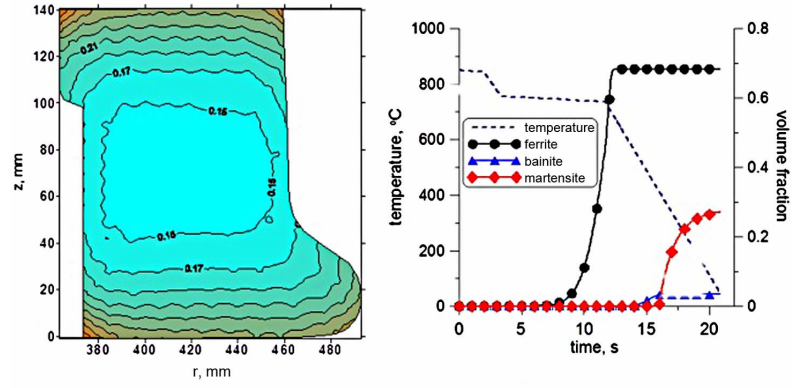

$$
\begin{gathered}
\text { conventional } \\
\text { model }
\end{gathered}
$$$$
\sigma_{p}=A \cdot \varepsilon^{B} \cdot \exp (C \cdot \varepsilon) \cdot \dot{\varepsilon}^{D+\frac{E}{T}} \cdot \exp \left(\frac{F}{T}\right)
$$
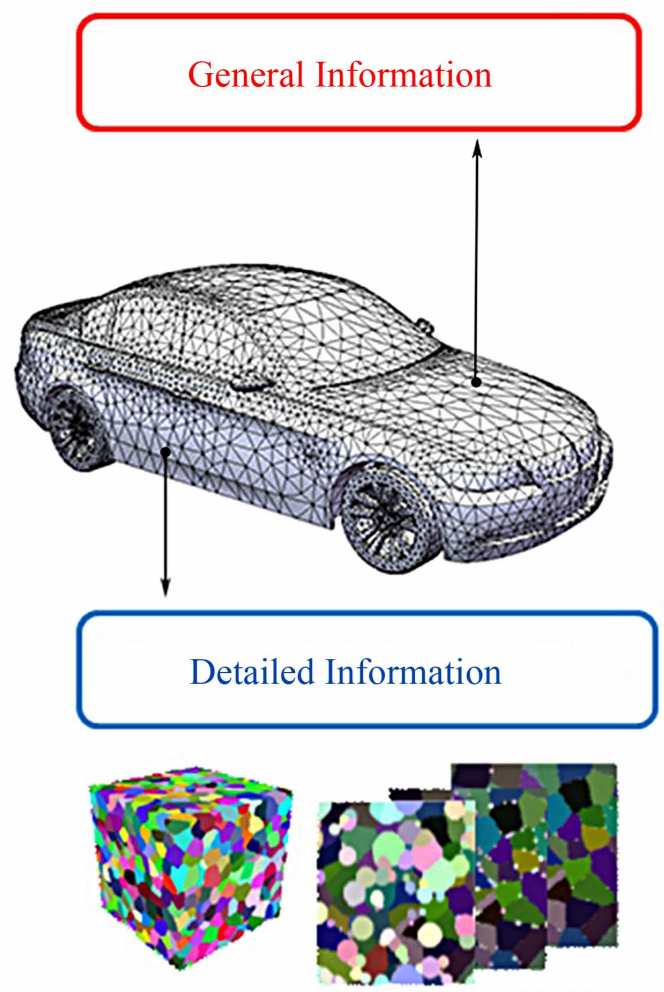

cellular automata model

Figure 1. Schematical illustration of advantages provided by the cellular automata material models.

able to each user. This allows focusing the main burden of the work on development of the appropriate transition rules that replicate physical phenomena and not on implementation aspects. As a result, the use of the framework to develop microstructure evolution models will be possible for material scientists without extensive knowledge of programming and algorithmic solutions. The CAF (Cellular Automata Framework) is based on the application-defined programming interface (Application Programming Interface, API), which allows for quick creation of a ready-made applications and reuse of developed codes. The framework was implemented based on the objectoriented technology, so that it was possible to make it in the form of the linked dynamic library for other developers and available interfaces which are shown in Figure 2. Details on this solution can be found in earlier authors work $[5,6]$.

Based on the Cellular Automata Framework functionality, the phase transformation model to predict changes from austenite to ferrite during continuous cooling was developed. Details of the model can be found in [7], while major assumptions and examples of obtained results are described in the following chapter.

\section{Description of Austenite-Ferrite Transformation Model}

The developed CA model is designed to simulate austenite to ferrite transformation during cooling in the 2D space. Each CA cell is described by several states and internal variables in order to properly describe physical state of the material. The cell can be in three different states (Figure 3):

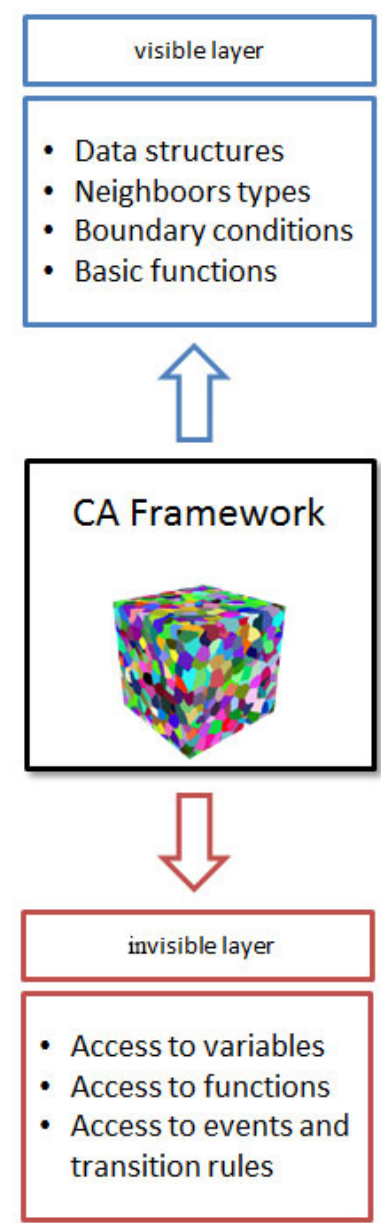

Figure 2. Interfaces of the CA framework. 


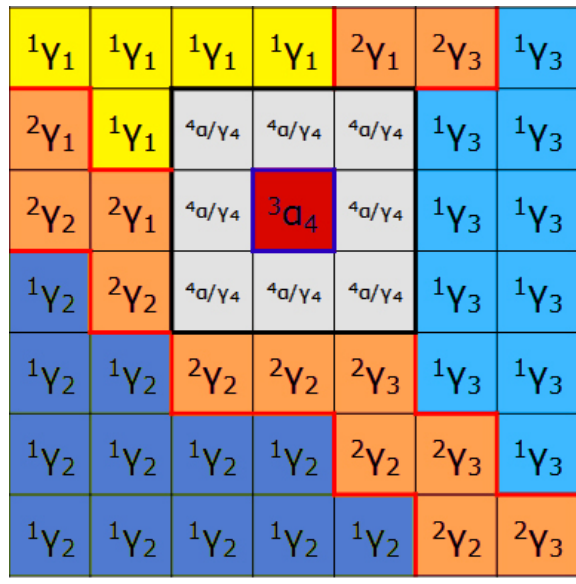

Figure 3. Illustration of the nucleus of the ferrite phase and the surrounding cells in the ferrite-austenite $(\alpha / \gamma)$ state.

- ferrite $(\alpha)$,

- austenite $(\gamma)$ and,

- ferrite-austenite $(\alpha / \gamma)$.

The last state describes CA cells located at the phase interface between austenite and ferrite grains. Additionally a series of internal variables were defined for each CA cell to describe other necessary microstructure features e.g. how many ferrite phase is in a particular cell $F_{i, j}$, what is the carbon concentration in each cell $C_{i, j}$, the growth length $l_{i, j}$ of the ferrite-austenite cell into the austenite cell or the growth velocity $v_{i, j}$ of the interface cell. These internal variables are then used in the transition rules to replicate mechanisms of phase transformation. Similar solutions with different level of complexity are also available in the literature [8,9].

The developed model takes into account two major mechanism occurring during transformation, namely nucleation and growth of the ferrite grains into the austenitic matrix. Due to the fact that nucleation process is considered as stochastic in nature, various approaches to describe this process can be used e.g. [8]. In the present model, to replicate the stochastic character of nucleation, a number of nuclei $n$ is calculated in a probabilistic manner at the beginning of each time step:

$$
\begin{gathered}
n=N\left(T_{i}\right)\left(\frac{B_{i}}{B_{0}}\right)^{3} P(\tau) \\
N\left(T_{i}\right)=\frac{a_{1}}{1+\exp \frac{\left(A_{e 3}-T_{i}\right)-a_{2}}{a_{3}}}\left(\frac{x y}{40000}\right) \\
P(\tau)=\frac{2}{3} \tau R(0,1)
\end{gathered}
$$

where: $B_{0}, B_{i}$ - mean amount of $\gamma$ cells at the beginning and $i$-th time step, respectively, $N$-total number of $\alpha$ nuclei, $a_{1}, a_{2}, a_{3}-$ model parameters, $A_{e 3}$ - start tempera- ture of the austenite - ferrite transformation, $x, y$ - width and height of the CA space, $P$-probability, $\tau$ - time step.

Also locations of grain nuclei in the CA space are also generated randomly along initial austenite grain boundaries. When a cell is selected as a nuclei, the state of this cell changes from austenite $(\gamma)$ to ferrite $(\alpha)$. At the same time all the neighboring cells of the ferrite $(\alpha)$ cell change their state to ferrite-austenite $(\alpha / \gamma)$ (Figure 3). The carbon content, which was in the austenite cell is then divided between all neighboring cells (the Moore neighborhood is used), which are in the state $\alpha / \gamma$. Nucleation process has a continuous character and it occurs during the entire CA simulation until the end of transformation. When a nucleus occurs in the CA space, the growth of ferrite phase is calculated in the following steps.

Ferrite growth is controlled by the carbon diffusion, thus the carbon distribution across the microstructure is evaluated by the solution of the diffusion equation on the basis of the finite difference (FD) method:

$$
\frac{\partial x_{\varphi}^{e}}{\partial t}=D_{\varphi} \nabla^{2} x_{\varphi}^{e}
$$

The transition rules describing growth of ferrite grains during phase transformation are designed to replicate experimental observations of mechanisms responsible for this process $[8,10]$. Because, newly formed ferrite nuclei grow into the austenite phase, the velocity of the $\alpha / \gamma$ interface is assumed to be a product of the mobility $M$ and the driving force for interface migration $F$ :

$$
v=M F=M_{0} \exp \left(-\frac{Q_{\text {act }}}{R T_{i}}\right) F
$$

where: $M_{0}$-mobility coefficient, $T$-absolute temperature, $Q_{\text {act }}$-activation energy.

The driving force for the phase transformation $F$ in the present work is based only on chemical driving force $F_{\text {chem. }}$. The driving force related with accumulated energy is neglected. The chemical driving force is a result of the differences in the carbon concentration in equilibrium conditions and carbon concentration in each cell:

$$
F_{\text {chem }}=\beta\left(C_{e q}\left(T_{i}\right)-C_{i, j}^{\gamma}\right)
$$

where: $\beta$-model coefficient, $C_{e q}$-equilibrium carbon concentration calculated using Thermocalc ${ }^{\mathrm{TM}}$ software, $C_{i, j}$ - carbon concentration in the $(i, j) \mathrm{CA}$ cell.

Equilibrium carbon concentration is calculated based on the Thermocalc ${ }^{\mathrm{TM}}$ data as schematically shown in Figure 4.

A set of transition rules is proposed in the model to replicate the phenomena occurring at the austenite-ferrite boundary. When the ferrite phase is present in the material, the CA ferrite cells grow into the austenite phase. In the current time step $t$ the growth length of the austen- 


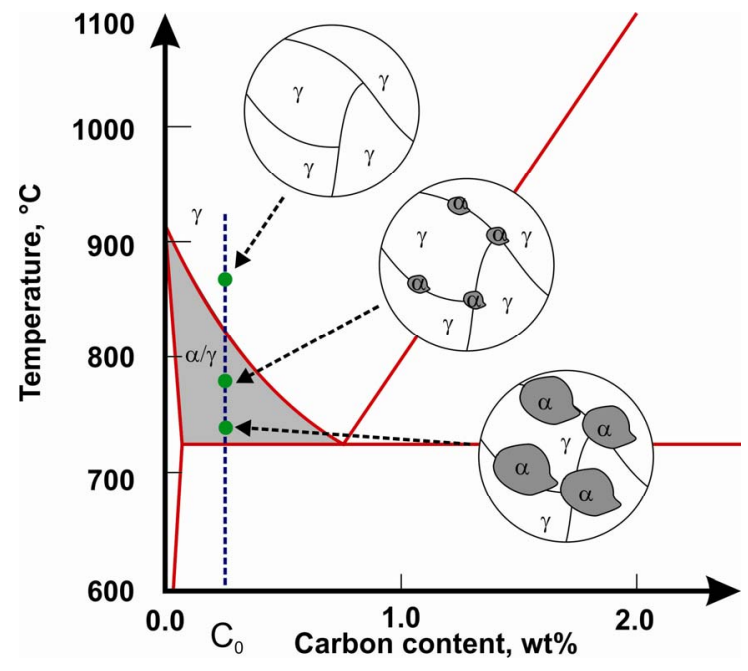

Figure 4. FeC equilibrium diagram.

ite-ferrite cell with indexes $(i, j)$ towards an austenite neighbouring cell with indexes $(k, l)$ is described as:

$$
l_{i, j}^{t}=\int_{t_{0}}^{t} v_{i, j} \mathrm{~d} t
$$

where: $t_{0}$ - time when the CA cell $(i, j)$ changed into the ferrite state, $v_{i, j}$ the growth velocity of the CA cell $(i, j)$.

The growth velocity $v$ is obtained from (5) and then the ferrite volume fraction in the CA cell $(k, l)$ is calculated as a result of the ferrite growth:

$$
F_{k, l}=\sum_{1}^{N_{\text {neigh }}} \frac{l_{i, j}^{t}}{L_{C A}}
$$

where: $F_{k, \Gamma}$ total ferrite volume fraction in the CA cell $(k, l)$, as a contribution from all the neighbouring austenite-ferrite CA cells, $L_{C A}$ - dimension of the CA cell in the CA space, $t$ - time step.

Based on these calculations the transition rules are defined as follows:

$$
\begin{gathered}
\mathrm{Y}_{k, l}^{t+1}=\left\{\begin{array}{l}
\alpha / \gamma \Leftrightarrow \mathrm{Y}_{k, l}^{t}=\gamma \wedge{ }^{M} \mathrm{Y}_{i, j}^{t}=\alpha / \gamma \wedge F_{k, l}^{t}>F_{c r} \\
\mathrm{Y}_{k, l}^{t}
\end{array}\right. \\
{ }^{N} \mathrm{Y}_{i, j}^{t+1}=\left\{\begin{array}{l}
t e m p \Leftrightarrow \mathrm{Y}_{k, l}^{t}=\gamma \wedge{ }^{N} \mathrm{Y}_{i, j}^{t+1}=\alpha / \gamma \\
\mathrm{Y}_{i, j}^{t}
\end{array}\right. \\
\mathrm{Y}_{k, l}^{t+1}=\left\{\begin{array}{l}
\alpha \Leftrightarrow \mathrm{Y}_{k, l}^{t}=\alpha / \gamma \\
\mathrm{Y}_{k, l}^{t}
\end{array} \rightarrow\right. \\
\mathrm{Y}_{k, l}^{t+1}=\left\{\begin{array}{l}
\alpha / \gamma \Leftrightarrow \mathrm{Y}_{k, l}^{t}=t e m p \\
\mathrm{Y}_{k, l}^{t}
\end{array}\right.
\end{gathered}
$$

where: $\mathrm{Y}_{k, l}^{t}$-state of the cell $(k, l)$ in the time step $t$, ${ }^{M} \mathrm{Y}_{i, j}^{t}$ - state of the Moore neighboring $(M)$ cell $(i, j)$ in the time step $t, F_{c r}$-critical value of the volume fraction of ferrite in the CA cell, temp - temporary state.
The CA cell changes the state from austenite into austenite-ferrite when ferrite volume fraction in this cell exceeds the critical value $F_{c r}$. Otherwise the cell remains in the austenite state see Equation (9). If the CA cell change state from austenite to austenite-ferrite state every von Neuman neighboring cells change state to temp state according to (10). After that transition rule described as (11) is initiated - every old austenite/ferrite cells change state to ferrite, and the cells on temp state change state to austenite-ferrite. When the cell changes its state to austenite-ferrite, all the neighboring cells in the austeniteferrite state change their states into the ferrite. When a change in the cell state occurs, the corresponding carbon concentration changes according to the $\mathrm{FeC}$ diagram. To obtain stable solution of the FDM and CA calculation and save computational time at the same time an effective time step refinement/derefinement procedure was implemented into the CAF.

Examples of obtained results on the basis of the model implemented in the CA framework are presented in Figure 5. Figure 5 shows the carbon distribution changes, corresponding phase fraction and microstructure morphology evolution during cooling with the cooling rate $1{ }^{\circ} \mathrm{C} / \mathrm{s}$. The size of the investigated CA space is assumed to be $200 \times 200$ cells what corresponds to the physical size $200 \times 200 \mu \mathrm{m}$.

As seen in Figure 5, the growth of newly formed ferrite nuclei is directly related with carbon concentration
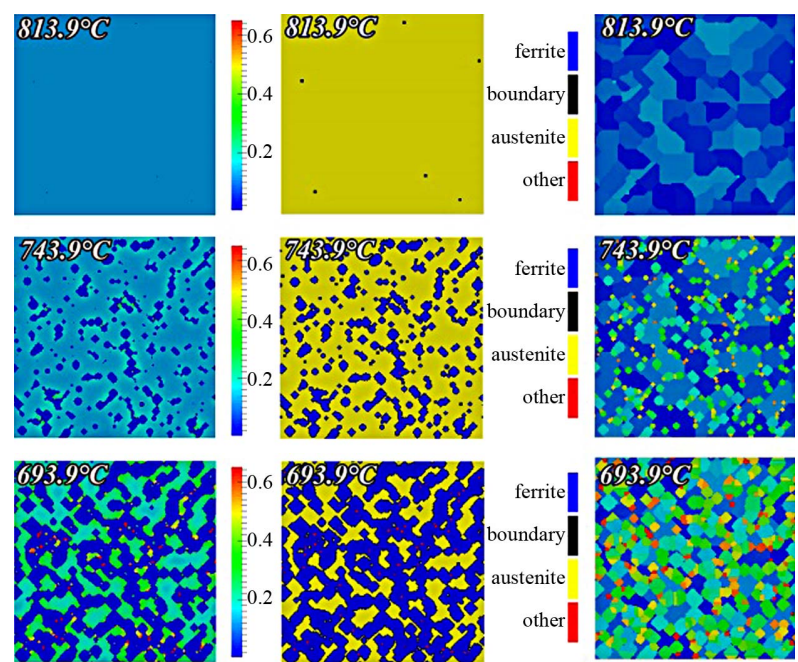

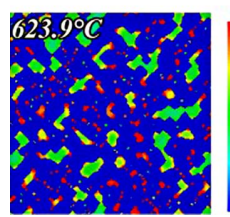

(a)

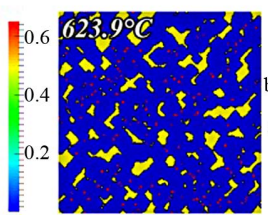

(b)

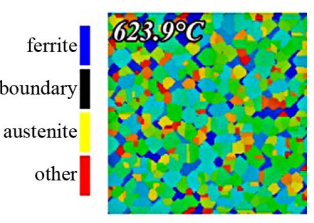

(c)
Figure 5. (a) Carbon distribution (b) phase fraction (c) microstructure morphology obtained during cooling with the cooling rate $1^{\circ} \mathrm{C} / \mathrm{s}$. 
controlled by the diffusion process.

As presented, developed model is based on several model coefficients that can directly influence quality of obtained results. Thus to evaluate level of influence of these model parameters on obtained results a sensitivity analysis based on Moris One At a Time (OAT) design was performed.

\section{Morris Oat Design}

The Morris design belongs to the class of screening methods [11]. Screening designs, as a part of the sensitivity analysis methods [12], deal with the question which factors of the physical model or computer simulation are really important. The factor means either parameter, which describes properties of the model or input variable, which is directly observable in the corresponding real system. Screening methods estimate qualitative statistic of the factors in order of their importance, i.e. they state that one factor is more important than another, but they do not provide the quantitative information of the factors significance.

Screening designs widely use the OAT approach. Methods based on the OAT technique investigate the impact of the variation of each factor in turn. The OAT design developed by Morris [13] is called the global sensitivity analysis, because the algorithm explores the entire space over which the factors vary. In this algorithm the main effect of the factor is estimated by computing the assumed number of local measures at different points in the input space and next the average value is taken. These points are selected in such a way that each factor covers the whole interval in which it was defined.

Sensitivity analysis was performed for 6 major parameters of the CA model: 3 parameters of nucleation model: $a_{1}, a_{2}, a_{3}$, mobility coefficient $M_{0}$, activation energy $Q_{a c t}$ and $\beta$ parameter. These parameters can be found in Equations (2)-(6). Additionally, to validate model functionality a 7 th parameter describing sensitivity to cooling rates was added to the investigation. Overall 800 calculations were realized during the investigation. Model output was defined as temperature of initiation and end of the transformation process.

As expected the model is sensitive to cooling rates, what proves physical nature of obtained results. It seems from Figure 6, that the most important model parameter is mobility coefficient that is directly related with the phase boundary movement. Thus, this coefficient has to be particularly well identified during the model identification procedure. Sensitivity of other model parameters remains on similar level.

\section{Conclusions}

Based on the presented research it can be stated that:

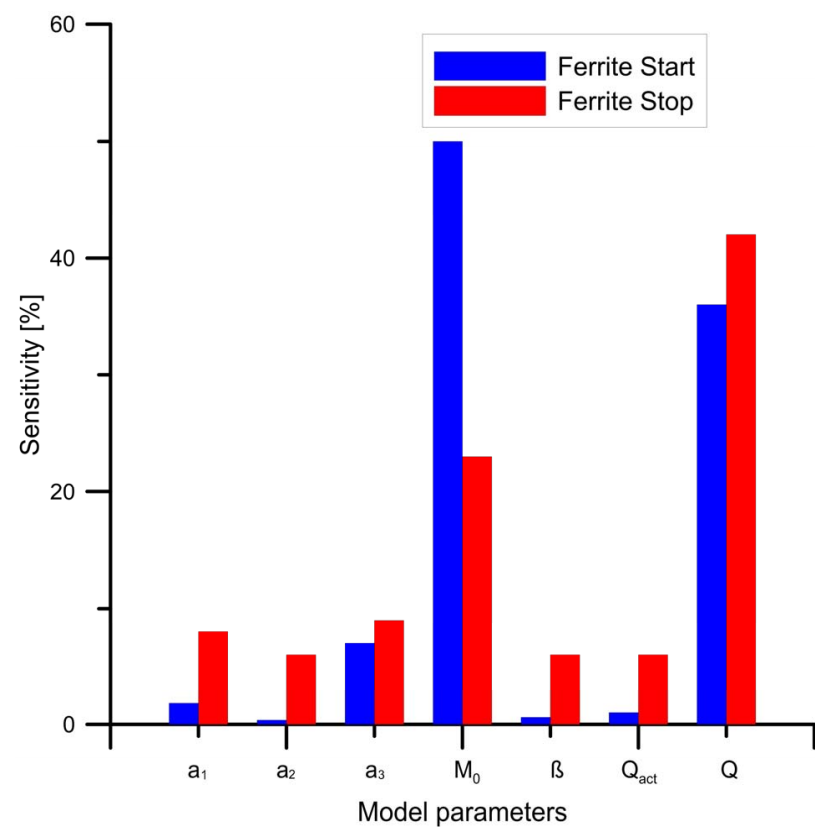

Figure 6. Sensitivity effect of different parameters for temperature ferrite start and ferrite stop.

- Implementation of the austenite-ferrite phase transformation model based on the developed CA framework is possible;

- The nucleation and growth parts of the model are directly related with changes in carbon concentration. Incorporation of the FDM into the CAF is a robust solution. The use of FDM for solving carbon diffusion provided a possibility for detailed reproduction of reality in the transformation of austenite-ferrite;

- Sensitivity analysis revealed that the model is mostly sensitive to the mobility coefficient and this parameter has to be carefully identified using inverse analysis. Sensitivity to other coefficients remains on similar level.

Future work will focus on identification of the CA model parameters based on inverse technique as well as further extension the CAF capabilities. Other microstructure evolution models e.g. recrystallization models will be also implemented and combined in the CAF using workflow approach.

\section{Acknowledgements}

Financial assistance of the NCN, project no. 2011/01/ D/ST8/01681, is acknowledged.

\section{REFERENCES}

[1] S. Das, E. J. Palmiere and I. C. Howard, "CAFE: A Tool for modeling Thermomechanical Processes," E. J. Palmiere, M. Mahfouf and C. Pinna, Eds., Proceedings of an International Conference on Thermomechanical Processing: Mechanics, Microstructure, Control, Sheffield, 23- 
26 June 2002, pp. 296-301.

[2] C. H. J. Davies, "Growth of Nuclei in a Cellular Automaton Simulation of Recrystalization," Scripta Materialia, Vol. 36, No. 1, 1997, pp. 35-40. http://dx.doi.org/10.1016/S1359-6462(96)00331-4

[3] J. Gawąd and M. Pietrzyk, "Application of CAFE Coupled Model to Description of Microstructure Development during Dynamic Recrystallization," Archives of Metallurgy and Materials, Vol. 52, No. 2, 2007, pp. 257-266.

[4] L. Madej, P. D. Hodgson and M. Pietrzyk, "Development of the Multi-Scale Analysis Model to Simulate Strain Localization Occurring during Material Processing," Archives of Computational Methods in Engineering, Vol. 16, No. 3, 2009, pp. 287-231. http://dx.doi.org/10.1007/s11831-009-9033-6

[5] P. Spytkowski, T. Klimek, L. Rauch and L. Madej, "Implementation of Cellular Automata Framework Dedicated to Digital Material Representation," Computer Methods in Materials Science, Vol. 9, No. 2, 2009, pp. 283-288.

[6] L. Rauch, L. Madej and K. Perzynski, "Numerical Simulations of the Microscale Material Phenomena Based on Cellular Automata Framework and Workflow Idea," Proceedings of the 17th ISPE International Conference on Concurrent Engineering, Cracow, 6-10 September 2010, pp. 539-546.

[7] R. Golab and L. Madej, "Development of the Complex Austenite-Ferrite Transformation Model Based on CA
Method," 22nd International Conference on Metallurgy and Materials, Brno, 15-17 May 2013.

[8] Y. J. Lan, D. Z. Li and Y. Y. Li, "Modeling Austenite Decomposition into Ferrite at Different Cooling Rate in Low-Carbon Steel with Cellular Automaton Method," Acta Materialia, Vol. 52, No. 6, 2004, pp. 1721-1729. http://dx.doi.org/10.1016/j.actamat.2003.12.045

[9] M. Pietrzyk, L. Madej, L. Rauch and R. Golab, "Multiscale Modeling of Microstructure Evolution during Laminar Cooling of Hot Rolled DP Steels," Archives of Civil and Mechanical Engineering, Vol. 10, No. 4, 2010, pp. 66-67. http://dx.doi.org/10.1016/S1644-9665(12)60031-4

[10] Y. J. Lan, N. M. Xiao, D. Z. Li and Y. Y. Li, "Mesoscale Simulation of Deformed Austenite Decomposition into Ferrite by Coupling a Cellular Automaton Method with a crystal Plasticity Finite Element Model," Acta Materialia, Vol. 53, No. 4, 2005, pp. 991-1003. http://dx.doi.org/10.1016/j.actamat.2004.10.045

[11] A. Saltelli, K. Chan and E. M. Scott, "Sensitivity Analysis," Wiley, Hoboken, 2000.

[12] M. Kleiber, H. Antunez, T. D. Hien and P. Kowalczyk, "Parameter Sensitivity in Nonlinear Mechanics," Wiley, Hoboken, 1997.

[13] M. D. Morris, "Factorial Sampling Plans for Preliminary Computational Experiments," Technometrics, Vol. 33, No. 2, 1991, pp. 161-174. http://dx.doi.org/10.1080/00401706.1991.10484804 